\title{
Crème de la Kremen of Wnt signalling inhibition
}

Ute Rothbächer and Patrick Lemaire

\begin{abstract}
Embryonic development depends on spatially regulated signals that function locally to change the fate or shape of cells. Recent advances have shed light on a sophisticated mechanism of Wnt signal inhibition, whereby an activating coreceptor, LDL-receptor-related protein 6 (LRP6), is segregated away by a secreted inhibitor (Dickkopf) and removed by internalization after interaction with the membraneanchored protein, Kremen.
\end{abstract}

$\mathrm{T}$ he signals mediated by secreted Wnt molecules are crucial during many phases of embryonic development. Perturbation of their signalling activity or their signalling pathway results in developmental defects or tumours ${ }^{1}$. Wnt interacts with the seven-transmembrane receptor Frizzled $(\mathrm{Fz})$, and this interaction is crucial for at least two distinct cellular processes ${ }^{2}$. The first process involves changes in cell fate, and this is achieved through an interaction of Wnt-Fz with the LRP6 coreceptor, a member of a family of molecules that are related to low density lipoprotein receptors (LDLRs). This ternary receptor complex formation is required to induce $\beta$-catenin accumulation and subsequent target gene expression, an event often referred to as the canonical Wnt or Wingless (Wg) pathway. The second process that can be induced by the Wnt-Fz interaction is a change in cell shape. This is achieved by Wnt-Fz activating the less well defined planar cell polarity (PCP) signalling pathway, which does not involve LRP6 (ref. 3) or $\beta$-catenin. Interestingly, although many of the intracellular mediators of Wnt signalling are conserved among metazoans (including hydra, insects and vertebrates), extracellular Wnt signal reception reaches a further level of regulation in chordates (for an overview, see http://www.stanford.edu/ rnusse/wntwindow.html). In this phylum, a number of secreted molecules that interfere with the binding of the Wnt signal through its receptor complex have been identified. Secreted molecules, such as secreted Frizzled-related proteins (sFRPs), Wnt-interacting factor (WIF) or Cerberus, are known to sequester the Wnt ligand and thought to block all Wnt signalling. Recent progress ${ }^{4}$ in understanding the mode of action of Dickkopf (Dkk), a second type of Wnt inhibitor that is thought to be specific for canonical Wnt signalling ${ }^{5}$, demonstrates the elaborate degree of complexity utilized in vertebrates to regulate if or how a cell is allowed to interpret the Wnt signal. Dickkopf not only means big-head, inspired from its overexpression phenotype in amphibians ${ }^{6}$, but also means stubborn in
German. Indeed, it seems that Dkk has its own way to inhibit Wnt signalling, a process that is required in normal head formation in Xenopus laevis and mice ${ }^{6,7}$. Dkk binds to and inactivates the LRP6 coreceptor ${ }^{5,8,9}$. Thus, Dkk regulates coreceptor availability rather than ligand availability. In a recent issue of Nature, Mao and colleagues reported findings that shed light on the mechanism of this coreceptor inactivation ${ }^{4}$. This study introduces a new player, the membrane-anchored molecule Kremen, which binds Dkk and triggers the internalization and clearing of the Dkk-LRP6 complex from the cell surface. The segregation and internalization of LRP6 renders Wnt-Fz unable to activate the intracellular $\beta$-catenin stabilization required for Wnt target gene expression (Fig. 1).

Kremen was identified in a transfection screen where pools of cDNAs were transfected into human cell lines and Dkk binding to cells was detected by enzymatic reaction. The ternary complex formation between LRP6, Dkk and Krm was shown elegantly in vivo through bioluminescence resonance energy transfer $(B R E T)^{10}$ experiments. The two membrane-anchored molecules were tagged with Renilla luciferase and yellow fluorescent protein (YFP), respectively, which 'light up' when activated and brought into proximity through the binding of both molecules to Dkk. Such methodology is expected to become an extremely useful tool in studying protein complex formation both outside and inside cells. Two related forms, Kremen1 and -2 (Krm1 and Krm2), were identified. Although several Dkk molecules also exist, the authors found that Dkk1 and Dkk2, but not Dkk3, bind with high affinity to both Krm1 and Krm2. Because Dkk1 blocks Wnt signalling, but Dkk2 and Dkk3 do not, this suggests a further level of control and raises new questions about their individual specificity. In some instances, Dkk2 may even potentiate Wnt signalling ${ }^{11}$. Therefore, in the future it will be important to define how the specificity is achieved in vivo.

LRP6 clearance was observed as a consequence of ternary complex formation in Krm- and LRP6-transfected cells provided with Dkk protein. After application of Dkk1, the membrane localization of tagged versions of LRP6 and Dkk1 diminished quickly. The proteins were instead subsequently enriched in intracellular vesicles, with a slight reduction in LRP6 levels. This decrease was interpreted as degradation and it will be important to verify this hypothesis with further studies. It will be especially interesting to determine whether $\mathrm{Krm}$ is being recycled to the cell surface on its own or whether it is also being degraded.

As neither $\mathrm{krm}$ nor $d k k$ genes can be clearly identified in the Drosophila melanogaster genome, whereas the LRP6 homologue arrow has been identified ${ }^{3}$, the authors used the fly as a reconstitution test tube to see whether the Krm-Dkk duo can be a similarly dangerous liaison in the fly. Indeed, when both $\mathrm{krm}$ and $d k k$ transgenes are expressed in wing precursor cells, aberrant wing formation is probably caused by decreased Wnt/Wg signalling. In support of this, they find that Dkk is actually bound by Arrow and functionally interacts with it in ectopic cell culture assays. This demonstrates how well-conserved the Wnt system is, even though all the components are not present in different model systems. Whether flies have functionally related Arrow-binding molecules or not is an open question. The main issue will now be to learn about the role of the endogenous Krm-Dkk-LRP interactions during embryonic development in chordates.

Wnt signal inhibition by LRP6 elimination through Dkk and Kremen is consistent with the findings that $\mathrm{Wnt}-\mathrm{Fz}$ is engaged in both canonical and PCP signalling and that LRP6 mediates canonical Wnt signalling only. Therefore, Dkk-1 and Kremen can be considered to be specific inhibitors of canonical Wnt signalling. Consistently, lossof-function phenotypes of Drosophila LRP6/Arrow ${ }^{3}$ do not have a PCP phenotype. Conversely, FRPs, which directly bind Wnt, inhibit both PCP and canonical signalling, reflected by their respective gain-of-function phenotypes in cell patterning versus defects in gastrulation movement ${ }^{5}$. Both 
Activation

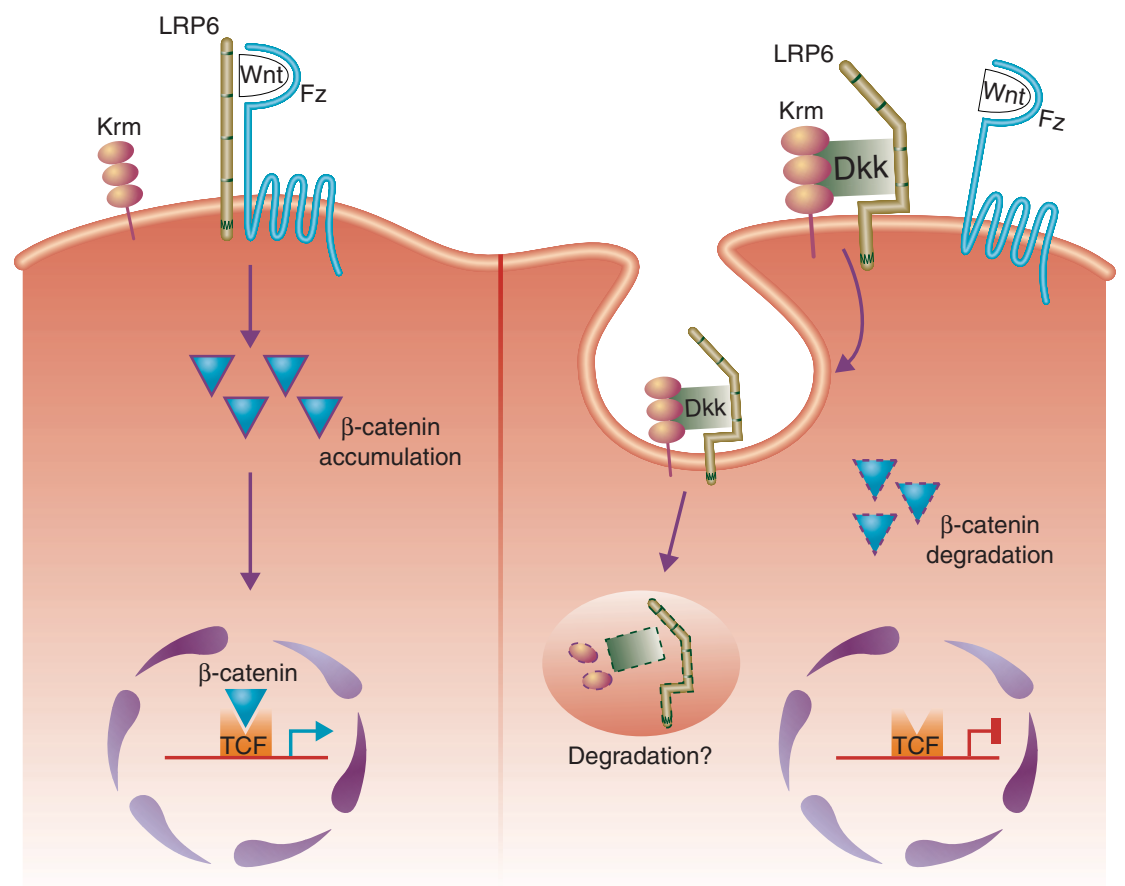

Figure $1 \mathrm{~A}$ model of canonical Wnt signalling inhibition through Kremen (Krm). a, In the absence of the inhibitor Dkk, the LRP6 coreceptor forms a complex with the Wnt ligand and the Fz receptor at the cell surface. This triggers accumulation of $\beta$-catenin and entry into the nucleus, activating target gene expression through the transcription factor TCF. b, In the presence of Dkk, ternary complex formation between Krm, Dkk and LRP6, and subsequent internalization in vesicles, results in depletion of LRP5/6 from the plasma membrane. Canonical Wnt signalling is blocked, $\beta$-catenin is not stabilized and undergoes degradation before it can allow gene expression in the nucleus.

Wnt signalling activities are mediated intracellularly by Dsh, which, through different domains, may transmit either signal to different molecular complexes ${ }^{12,13}$. Interestingly, Dsh may associate with Axin, an important factor of the $\beta$-catenin degradation complex that is considered to be specific for the canonical pathway because of a lack of PCP phenotype in fly mutants ${ }^{14}$. In this context, it is important to note that Axin was also found to bind directly to the LRP5 intracellular domain ${ }^{15}$. This raises the possibility that the internalization of LRP5/6 into degradation vesicles also affects functions of Axin. It will therefore be essential to determine what the consequences of Krm-Dkkinduced LRP internalization are on the degradation of the Axin- $\beta$-catenin complex.

Three conserved extracellular domains in Kremens are related to defined domains in other proteins, and all are indispensable for Dkk binding. Membrane attachment is mediated by a carboxy-terminal transmembrane stretch and this seems crucial for Wnt inhibition. Interestingly, the transmembrane and cytoplasmic domain can be substituted by a glycosyl phosphatidylinositol (GPI) anchor, suggesting that the sole function of the $\mathrm{C}$ terminus in the clearance process is membrane anchoring. It will be challenging to find out how Kremen can trigger internalization of LRP6, as Arrow/LRP6 itself contains an internalization consensus in its cytoplasmic tail ${ }^{3}$. LRP5 and LRP6 are both related to LDLRs, several of which are known to self-internalize and remove extracellular ligands ${ }^{16}$. The function of Kremen may be to allow LRP6 clearance by unmasking the putative LRP6 internalization signal. Selectively mutating the LRP6 motif and testing its effect on internalization should determine whether a block of Wnt signalling by Dkk/Krm-mediated LRP6 clearance resembles known ligand-mediated LDLR internalization.

Endocytosis has previously been suggested to restrict Wg activity at parasegmental borders in the Drosophila cuticle ${ }^{17}$. In this case, the Fz receptor is thought to contain an endocytic signal for Wnt ligand downregulation. At first sight, this process does not seem to be related to the described LRP6 clearance that blocks the Wnt signal, but it will be interesting to test whether Arrow is involved in this process. Conversely, the Dkk-LRP6 interaction was suggested to disrupt the LRP6-Wnt-Fz ternary complex $^{5}$, suggesting that LRP6, but not Wnt and $\mathrm{Fz}$, is internalized. However, this study used only the extracellular domains of receptors and did not determine whether LRP6-Wnt-Fz complex formation is disrupted in vivo. Krm-induced internalization should therefore be analysed in relation to the other members of extracellular and intracellular components of the Wnt receptor complex, such as Wnt and Fz, but should also include Axin and Dsh. This should provide a better picture of how these various partners are affected by LRP6 clearance.

Wnt signalling has taught us to reject all simplistic concepts of a growth factor binding to its receptor to activate a linear intracellular pathway that triggers target gene expression in the nucleus. We now know that each level of extracellular or intracellular signal propagation depends on a mass of molecular interactions that decide how a specific signal is finally interpreted within the cell. Internalization of the ligand and/or the receptor into degradation vesicles adds a new degree of complexity and provides a sophisticated way to limit signalling in the presence of the ligand. Most likely, we can expect that many more of these subtle tricks will be discovered in a variety of other signalling cascades.

Ute Rothbächer* and Patrick Lemaire $\dagger$ are in the Institut de Biologie du Développement de Marseille, Laboratoire de Génétique et Physiologie du Développement, CNRS Université de la

Méditerranée, Case 907, Campus de Luminy, F13288 Marseille, France

${ }^{*}$ e-mail: rothbach@lgpd.univ-mrs.fr

†e-mail: lemaire@lgpd.univ-mrs.fr

1. Huelsken, J. \& Birchmeier, W. Curr. Opin. Genet. Dev. 11, 547-553 (2001).

2. Niehrs, C. Nature $413,787-788$ (2001).

3. Wehrli, M. et al. Nature 407, 527-530 (2000).

4. Mao, B. et al. Nature 417, 664-667 (2002).

5. Semenov, M. V. et al. Curr. Biol. 11, 951-961 (2001).

6. Glinka, A. et al. Nature 391, 357-362 (1998).

7. Mukhopadhyay, M. et al. Dev. Cell 1, 423-434 (2001).

8. Bafico, A., Liu, G., Yaniv, A., Gazit, A. \& Aaronson, S. A. Nature Cell Biol. 3, 683-686 (2001).

9. Mao, B. et al. Nature 411, 321-325 (2001).

10. Xu, Y., Piston, D. W. \& Johnson, C. H. Proc. Natl Acad. Sci. USA 96, 151-156 (1999).

11. Wu, W., Glinka, A., Delius, H. \& Niehrs, C. Curr. Biol. 10, 1611-1614 (2000).

12. Axelrod, J. D., Miller, J. R., Shulman, J. M., Moon, R. T. \& Perrimon, N. Genes Dev. 12, 2610-2622 (1998).

13. Wallingford, J. B. et al. Nature 405, 81-85 (2000).

14. Hamada, F. et al. Science 283, 1739-1742 (1999).

15. Mao, J. et al. Mol. Cell 7, 801-809 (2001).

16. Herz, J. Trends Neurosci. 24, 193-195 (2001).

17. Dubois, L., Lecourtois, M., Alexandre, C., Hirst, E. \& Vincent, J. P. Cell 105, 613-624 (2001). 